\title{
(6) \\ OPEN ACCESS \\ Development of a tool for defining and identifying the dying patient in hospital: Criteria for Screening and Triaging to Appropriate aLternative care (CriSTAL)
}

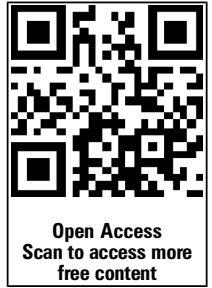

- Additional material is published online only. To view please visit the journal online (http://dx.doi.org/10.1136/ bmjspcare-2014-000770).

${ }^{1}$ The Simpson Centre for Health Services Research, South Western Sydney Clinical School, The University of New South Wales, Kensington, NSW 2052, Australia

${ }^{2}$ The Simpson Centre for Health Services Research, South Western Sydney Clinical School, The University of New South Wales \& Liverpool Hospital, Liverpool BC 1871, New South Wales, Australia

\section{Correspondence to} Dr Magnolia Cardona-Morrell, The Simpson Centre for Health Services Research, South Western Sydney Clinical School, Level 1, AGSM Building, The University of New South Wales, Kensington NSW 2052, Australia;

m.cardonamorrell@unsw.edu.au

Received 31 July 2014 Revised 23 October 2014 Accepted 23 November 2014

CrossMark

To cite: Cardona-Morrell M, Hillman K. BMJ Supportive \& Palliative Care 2015;5:78-90.

\section{Magnolia Cardona-Morrell, ${ }^{1}$ Ken Hillman ${ }^{2}$}

\section{ABSTRACT}

Objective To develop a screening tool to identify elderly patients at the end of life and quantify the risk of death in hospital or soon after discharge for to minimise prognostic uncertainty and avoid potentially harmful and futile treatments.

Design Narrative literature review of definitions, tools and measurements that could be combined into a screening tool based on routinely available or obtainable data at the point of care to identify elderly patients who are unavoidably dying at the time of admission or at risk of dying during hospitalisation.

Main measurements Variables and thresholds proposed for the Criteria for Screening and Triaging to Appropriate aLternative care (CriSTAL screening tool) were adopted from existing scales and published research findings showing association with either in-hospital, 30-day or 3-month mortality.

Results Eighteen predictor instruments and their variants were examined. The final items for the new CriSTAL screening tool included: age $\geq 65$; meeting $\geq 2$ deterioration criteria; an index of frailty with $\geq 2$ criteria; early warning score $>4$; presence of $\geq 1$ selected comorbidities; nursing home placement; evidence of cognitive impairment; prior emergency hospitalisation or intensive care unit readmission in the past year; abnormal ECG; and proteinuria.

Conclusions An unambiguous checklist may assist clinicians in reducing uncertainty patients who are likely to die within the next 3 months and help initiate transparent conversations with families and patients about end-of-life care. Retrospective chart review and prospective validation will be undertaken to optimise the number of prognostic items for easy administration and enhanced generalisability. Development of an evidence-based tool for defining and identifying the dying patient in hospital: CriSTAL.

\section{BACKGROUND}

The natural progression of chronic disease involves periods of apparent remission interspersed by exacerbations and, in the year leading to death, multiple hospitalisations. ${ }^{1}$ Some indicators of poor prognosis can suggest a patient is nearing the end of life, ${ }^{2}$ and have been found useful for initiating discussions with families regarding pre-emptive care planning. ${ }^{1}$ Yet there is uncertainty of the time, frequency and duration of the next episode of decompensation as well as the ultimate prognosis causing doubts about whether to continue active management. Further, while the majority of people want to die at home, most will die in hospital. ${ }^{3-8}$ Patients nearing the end of life are high-level users of ambulance services, ${ }^{9}$ emergency services, $^{2}{ }^{10}$ hospital wards ${ }^{11}$ or intensive care units and many die in hospital. ${ }^{12}$ Significant numbers of patients with cancer or other terminal illnesses are suitable for palliative care but often are readmitted to acute hospitals multiple times with lengths of stay of just under a week. ${ }^{10} 13{ }^{14}$ While there are accepted policies for de-escalating treatment in terminally ill patients, ${ }^{2}$ 15-17 there are also inherent and societal pressures on medicine to continue utilising technological advances to prolong life even in plainly futile situations. ${ }^{15}$

The implications of a decision to administer or withhold aggressive treatment for terminal patients are enormous for clinicians, patients and their families, for the health system and for society as a whole. It can be difficult to reach a decision that balances the rights of patients to die with dignity $^{18} 19$ and the expectations of families about satisfactory end-of-life care, ${ }^{20} 21$ 
while considering the limitations of health resources where opportunity costs cannot be disregarded. ${ }^{22-24}$ Delaying unavoidable death contributes to unsustainable and escalating healthcare costs, despite aggressive and expensive interventions. These interventions may not influence patient outcome; often do not improve the patient's quality of life; may compromise bereavement outcomes for families; and cause frustration for health professionals. ${ }^{20} 25-29$ This highlights the importance of developing more accurate ways of identifying patients near the end of life, involving both the patients and their carers in those discussions and then making more appropriate management plans.

For about two decades many acute hospitals have adopted rapid response systems to identify and manage seriously ill patients. ${ }^{25}$ 30-32 They were initially developed to recognise at-risk patients early as a basis for triggering a rapid response to improve patient outcomes. In doing so, the system also identifies patients at the end of life who are predictably deteriorating. Up to one-third of all of rapid response team (RRT) calls have been related to end-of-life issues. $^{33} 34$ This emphasises the failure of current hospital systems to recognise patients at the end of life. Often it is the patient and carers who initiate this conversation. $^{25} 35$

Clinical decision aids are widely used to involve patients in informed treatment decisions that incorporate their personal preferences and values. ${ }^{36}$ Sensitive clinical decision rules have been used to discontinue futile resuscitation on patients who experience a cardiac arrest. ${ }^{37}$ However, we have not found a fit-for-purpose screening checklist or clinical decision tool for objective identification of end of life within days, weeks or months to minimise inappropriate treatment at hospital admission. ${ }^{29}$ There is a need to recognise patients at the end of life while at the same time acknowledging uncertainty around the exact time and circumstances when death will occur. ${ }^{38}$

The aims of the CrisTAL checklist are to assist clinicians to recognise these patients and to change the culture of the hospital to one where end of life is openly discussed and dealt with more appropriately. ${ }^{39}$

\section{RATIONALE}

Accordingly, there is a need to collate evidence to assist clinicians, carers and families in decision-making about the most sustainable model for appropriate and best quality care in the last few months of life. The specific objectives of this research are to:

1. review literature to obtain definitions for dying patient and end of life;

2. review existing literature regarding screening tools for the prediction of death in hospitalised patients;

3. propose a checklist for screening of hospitalised patients at-risk of dying in the short to medium term.

Two common and important situations where patients at the end of life can potentially be identified are on admission to the emergency department (ED); and when a patient deteriorates and becomes the subject of a RRT call. This paper reports on the development of a clinical decision aid for use in both circumstances: CrisTAL (Criteria for Screening and Triaging to Appropriate aLternative care). It summarises the information available in the literature to construct the domains for such a screening instrument based on patient data items routinely available at the point of care.

The tool is intended to offer a starting point to begin discussions with the patient and relatives about priorities and preferences on type and place for end-of-life care. ${ }^{39}{ }^{40}$ It also may identify elderly who will benefit from alternative care pathways instead of hospitalisation. ${ }^{541}$ The routine use of such a tool may also change the culture of the organisation to one which is more aware of patients who may be at the end of life and one where different management pathways are considered earlier. The tool is not meant to dictate whether or not a patient receives life sustaining therapy or is the subject or a do-not-resuscitate order. However, it may provide an objective assessment to inform and support that decision, made jointly by patients, their family and the treating team.

\section{METHODS}

We undertook a narrative literature search in PubMed, Cochrane Library and Google Scholar for published and unpublished papers about explicit and practical definitions of 'end of life' and for tools or screening instruments to predict death. The search strategy included the following terms: (End of life, terminal, dying, inappropriate resuscitation, do-not-resuscitate, cardiopulmonary resuscitation order, limitations of treatment, discontinuation of care, futility, advanced directive) and (hospital, acute care facility, palliative care, ED) and (Screening tool, decision aid, algorithm, predictive, predictor of death). This was supplemented with manual searches through the reference lists of eligible papers.

The variables and thresholds explored for the screening tool were adopted from existing scales and published findings that demonstrated their association with either in-hospital or 30-day mortality or survival to 12 weeks. ${ }^{4-47}$ Based on the practicalities of applying the tool as decision-making support at the point of care, we used four criteria to decide whether the existing instrument was helpful for the purpose of objectively diagnosing dying and whether to discard items: ready availability in medical records, ${ }^{42}{ }^{43}$ need for clinical judgement, use of value judgment and self-sufficiency of indicators. This review was followed by consultation with two doctors and three ICU nurses with intensive care qualifications and experience in end-of-life care, about the feasibility of acquiring or documenting these data items in routine care. 


\section{RESULTS}

We found 112 relevant articles dealing with the definition of dying, determination of severity of deterioration, prediction of in-hospital death, preferred place of death and options for alternative end-of-life care. Among these, we identified 18 instruments and their variants validated in different settings. Below is a summary of the operational definitions and commonly used or cited tools to predict death in hospital.

\section{Operational definitions}

Nine working definitions of end of life were found to assist in limiting the number of items for a screening tool to a manageable set (table 1). These were mostly impractical in their requirement of clinicians' subjective assessment; or confined to patients imminently dying within hours; and of limited use for elderly patients with chronic disease, nearing end of life within days or weeks.

We defined inappropriateness of admission to hospital for patients at the end of life as those 'admissions when the resources of the hospital will not have any significant impact on the clinical prognosis of the elderly patient with multiple life-threatening comorbidities'.

As pragmatic definitions of 'dying patients' were not prevalent in the literature, we searched for a suitable

\section{Table 1 Definitions of end of life and their suitability for routine use in screening}

\begin{tabular}{lll} 
Year & reference & Definitions or potential items to include in a definition \\
\hline 1981 & $\begin{array}{l}\text { US President's } \\
\text { Commission }\end{array}$ & $\begin{array}{l}\text { When a terminally ill, mentally competent patient refuses } \\
\text { resuscitative treatment and/or where treatment would be futile }\end{array}$
\end{tabular}

Comments and rationale for inclusion or exclusion Does not assist in applying terminology in a screening tool for use in routine care as it would be impractical without operational boundaries or classification of irreversible conditions or futile treatments

1987 Blackhall ${ }^{49}$

1989 Stolman ${ }^{15}$

1990 Tomlinson and Brody 50

2005 Paterson, UK. ${ }^{41}$

2006 NHMRC $^{51}$

2007 NICE, UK 21

2007 Jones et a/ ${ }^{35}$

2014 Schmidt and Moss $^{52}$
When treatments will not be beneficial and may even be potentially harmful

Terminally ill patient, imminently dying (life expectancy $\leq 6$ months) chronic debilitating irreversible condition where life-saving treatment would be futile. Coupled with competent patient with unacceptable quality of life who refuses treatment or whose family requests to not resuscitate

When treatment is futile, defined as intervention (such as CPR) on terminal cases that provide no physiological benefit to the patient, that is, restoring spontaneous heart beat or blood pressure

Patients expected to die within 24 hours are those who were unconscious, self-ventilating, deteriorating and having a diagnosis incompatible with survival

Patients requiring frequent intervention, being bed-bound, irreversible loss of appetite, profound weakness, trouble swallowing, dry mouth, weight loss, becoming semiconscious, with lapses into unconsciousness, and experiencing day-to-day deterioration that is not reversible

Group 1: 'those with advanced, progressive, or incurable conditions who are expected to die within the next 12 months', and

Group 2:'adults with existing conditions who are at risk of dying from a sudden, acute crisis in their condition'; this group includes those with life-threatening acute conditions caused by sudden catastrophic events

Elderly with multiple-pre-existing comorbidities and mostly designated NFR at the time of death (pre-existing or newly designated) with or without evidence of advanced care planning

Patients suffering from poor quality of life due to clinical deterioration that is subtle and not immediately life-threatening but in whom the burden of treatment substantially outweighs the benefit
This concept may be clearer for specialists but not so useful for first-line doctors/nurses at admissions in ED

Life expectancy would require a prognostic table and some patients with low quality of life may refuse treatment but they are not imminently dying

While philosophically sound, it clearly involves clinical and value judgment that could vary from one clinician to another

This framework for end-of-life care was introduced to help clinicians in the delivery of care for the acutely dying, that is, who should not be triaged if they are at that advanced stage in the dying process at the time of admission

Combination of objective signs and symptoms and subjective considerations to be used in routine practice as indications of an imminent death; suitable for use in nursing homes and may more closely fit the needs at the hospital admission department for identification of patients dying over the next few days but does not cover the profile of those dying over weeks or months

Our manuscript is concerned with the first group, where the prediction of time to death is more feasible, but the definitions above are still not operative due to the uncertainty and dependency on expertise of subjective clinical or value judgements

This is a minimum standards definition applying to a well-defined patient group that triggers a RRT call; This represents the readily identifiable tip of the iceberg. We are also seeking to target those other patients with undiagnosed organ failures and without a documented NFR orders at the time of presentation to hospital for end-of-life screening so they can be offered end-of-life care out of acute hospitals

Conceptually encapsulates the definition of dying in the short term but it is difficult to measure without a checklist or classification as it involves clinical and value judgements which leave room for interpretation among healthcare professionals 
proxy measure that could be drawn from studies examining predictors of poor survival. These are abundant and cover both subjective and objective parameters anticipating death.

\section{Subjective variables and their utility in predicting short-term to medium-term mortality}

Of the instruments developed in the past 30 years for prognostication of death after admission, many still require value judgements and unstructured subjective assessments, which renders them less reassuring and hence less useful as a tool for deciding at the time whether to admit a patient.

Performance Status Scales designed as early as 1949 by Karnofsky ${ }^{53}$ and The World Health Organisation (ECOG PS) in 1982 are simple and popular instruments for determining appropriate intensity of care for patients. They have undergone adaptations over time ${ }^{55}$ where completion still involved major value judgements, which makes them impractical and unreliable for a standardised prognosis (table 2).

Various indices have been designed to identify illness severity and risk of death after admission (table 2). Some reliably capture the level of quality of life in terminal patients but do not focus on objective signs $;{ }^{54}$ some use nursing assessment of organic and psychosocial aspects $;^{56}$ others suggest a checklist that combines objective (eg, semiconsciousness) and subjective items (eg, 'irreversible deterioration'). ${ }^{51}$ Some emphasise application of survival prediction for in hospital-based palliative care services with high prognostic accuracy $(85.6 \%)$ in estimating death within 3 days of admission to a palliative care facility, but only $54 \%$ and $57.6 \%$ accuracy in predicting death within 4-30 days and by 6 months. ${ }^{57}$

A global assessment of frailty using a subjective score between 1 (very fit) and 7 (severely frail) had good predictive validity for death within 18 months ${ }^{58}$ but required clinical and value judgements, and did not incorporate the impact of underlying conditions, hence reducing its ease of use for routine care by less experienced personnel. Clinician perception about risk of death has been found to be reasonably accurate in particular for patients with advanced chronic heart failure or chronic obstructive pulmonary disease as an adjuvant in the decision to more efficiently target palliative services and end-of-life care planning. ${ }^{59}$

Finally, the global self-rated question designed to assess patient perception of their own health in comparison with other people their age, not intended as a prognostic tool but since the early 1980s has been associated with predicting long-term mortality in the elderly, ${ }^{60}$ independently of 'objective health status' and across ethnic groups. The self-rated health question is now better understood, ${ }^{60-62}$ and has been validated as a screening tool for vulnerable people at higher risk of death in community. ${ }^{63}$ Its influence on imminent risk of death at hospital admission is not known and we will include it in our screening tool.

\section{Objective variables and their utility in predicting short-term mortality}

Several factors have been found to have an impact on the risk of death after hospital admission, including age 65 years and above, ${ }^{42638586}$ multiple comorbidities, ${ }^{18}$ multiorgan failure, ${ }^{44}$ physiological data from laboratory test results ${ }^{64} 76$ and type of service and urgency of admission. ${ }^{43}$

We propose a combined algorithm quantifying the aggregate risk estimation of some previously developed instruments to take us closer to a more accurate definition of dying. An historical exploration of 18 of these estimates has shed more light on the influence of these factors.

The diagnosis of advanced cancer has probably attracted the most attention for predicting prognosis and appropriate care. From a review of 24 studies and 18 prognostic indicators, there was general agreement that anorexia and weight loss showed the most significant association with poor survival, followed by cognitive impairment, dyspnoea and dysphagia. ${ }^{83}$ While several of these studies were conducted in small convenience samples, some with doubtful statistical methods, ${ }^{42}$ clinicians would agree that these are largely symptoms of imminent death. Uncertainty of what constitutes dying in the short term has led to the development of practical prediction tools to assist in treatment decision-making, guide family consultations, and minimise unnecessary expense to the health system (table 2).

\section{Prognostic scales and indices}

Performance Status Tools have been well received and modifications tested in various settings.

Table 2 summarises scales found a predicting outcome and time to death/discharge, some of which have been validated in similar or divergent populations and others have led to refinements and developments of further tools. ${ }^{72}$ many are cancer-specific scales, thus have limited value for wider use in ED. ${ }^{82}$ For instance the $\mathrm{PaP}$ score is good at reducing the prognostic uncertainty of death within 1 month of admission to palliative care services. ${ }^{76}$ However, it is only validated for patients with cancer and it can yield significant differences between the prediction of registered nurses and doctors. ${ }^{76} 7787$

The Charlson Comorbidity Index (CCI), was designed to estimate 1-10-year mortality in longitudinal studies and is not validated as prognostic indicator for short-term outcomes in cancer or other conditions. $^{88}$ The Elixhauser Comorbidity Index is a complex tool $^{74}$ which uses administrative databases to estimate increased risk of in-hospital death or prolonged hospital stay. ${ }^{68}$ but clinicians may not find it user-friendly because it relies on administrative data 
Table 2 Existing scales or screening tools to predict risk of death and their domains

1998 Elixhauser ${ }^{73}$ 2009 Van Walraven ${ }^{18}$ 2013 Austin $^{74}$

\section{Subbe $^{75}$}

2004 Glare $^{76}$

2012 Maltoni $^{77}$

2013 Kuo-H $^{79}$

2005 Rockwood $^{58}$

2006 Paterson $^{78}$

SEWS Index Scale
Acute Physiology and

Chronic Health

Evaluation

APACHE ॥

APACHE III

APACHE IV

APACHE-L

Charlson Comorbidity Index (CCI)

SAPS ॥

Palliative Performance Scale (PPS)

Elixhauser comorbidity

MEWS

Palliative Prognostic Score (PaP)

Rapid Emergency Medicine Score (REMS)

CSHA Clinical Frailty the basis of qualitative definitions incorporating physical functioning and presence of comorbidities

Respiratory rate, oxygen saturation, temperature, blood pressure, heart rate and conscious leve

rate

Respiratory rate, Sodium (serum)

Haematocrit, White cell count,

lades 19 categories of comorbidity and ach condition is assigned with a severity score of 1, 2, 3 or 6 depending on the greater comorbidity (patients with a score $>5$ have a $100 \%$ risk of dying at 1 year)

Age, heart rate, Systolic BP,

Temp, GCS, CPAP Y/N, PaO, $\mathrm{FIO}_{2}$, urine surgical admission

Assessment of observed ambulation, activity, evidence of disease self-care, intake, level of physical activity and level of consciousness.

Score $0=$ death

Score $70=$ bed bound

Score $100=$ full health and ambulation

Karnofsky Performance Status plus

Dyspnoea

Anorexia

Clinician's weighted prediction of survival

Blood pressure, respiratory rate, Glasgow Coma Scale, peripheral oxygen saturation
CrisTAL Inclusion criteria and comments

$\begin{array}{llll}\begin{array}{l}\text { Readily } \\ \text { available }\end{array} & \begin{array}{l}\text { Clinical } \\ \text { judgement }\end{array} & \begin{array}{l}\text { Value } \\ \text { judgement }\end{array} & \begin{array}{l}\text { Sufficient for } \\ \text { prognosis }\end{array} \\ \square & \square & \checkmark & \square\end{array}$

Administered face to face or by phone; involves value judgements; poor inter-rater reliability; does not cater for preadmission functional status

$\square$
High clinician's acceptability as it takes 1 min to administer but has not proven accurate in predicting death within 6 months in individuals

$\square \quad \square \quad \square \quad \square$ Used to predict hospital mortality in ICU. Unsuitable for admissions unit risk of dying associated with this condition. Higher scores indicate

Scores of 5 or more were associated with increased risk of death

Data items which are incomplete and not detailed enough to
Complex calculation. Many adaptations attempted to improve predictive accuracy of 10 -year mortality. Some capability for predicting short-term mortality. Does not cater for functional status or immediate risk of death, that is, physiological risk

Validated in 12 countries and the results were encouraging even in the absence of a primary diagnosis but high reliance on sophisticated testing not routinely conducted outside ICU

$\square$ $\square$

Validated in Canada and Australia. However, the original intention of developers was not to use PPS for prognostication. ${ }^{71}$ Subjective observations do not contribute to standardisation of assessment

Recent validation showed a PPS of 10 was associated with over $90 \%$ in-hospital mortality whereas a PPS of 70 was associated with $0 \%$ deaths provide a clinically precise time of diagnosis. Complex to calculate, not too accurate on predicting mortality, more useful for researchers than clinicians at predicting length of stay

$\begin{array}{llll}\square & \square & \square\end{array}$

Good predictive ability for risk of death in busy acute services $\begin{array}{lccc}\square & \square & \square \\ \text { Validated in Italy, Australia and England. Good association }\end{array}$ with short-term mortality but predictive value of tool affected by less experienced doctors

$\square \quad \square$

Effective in predicting risk of death in hospital in conjunction with other clinical parameters including surgical treatment within $24 \mathrm{~h}$. However, it has little relevance for elderly patients with chronic disease seeking hospital care

\section{Each 1-category increment of the frailty scale increased the} risk of mortality. Largely subjective or reliant on clinical and value judgements

$\square$ $\square$ $\square$

Score correlated both with in-hospital mortality and length of stay 
involved clinical judgements and one involved value judgment; nine tools met two criteria and four tools only met one criterion. Figure 1 shows the distribution of criteria to justify the need for a fresh tool that met the four criteria.

In the absence of a comprehensive instrument combining acute and chronic predictors to increase certainty of diagnosis of imminent death or death within weeks or months, this review gathered recognised predictors of death for elderly patients with complex health profiles from existing prognostic tools to create a new screening instrument. We anticipated that incorporating objective variables would enhance certainty of the screening tool and could assist in the decision to appropriately generate do-not-resuscitate orders $^{25}$ and consider alternative end-of-life care orders.

The variables and values proposed for the CriSTAL screening tool were adopted from existing scales and from published research findings demonstrating their association with either in-hospital or 30-day mortality or survival to 12 weeks. ${ }^{42-45} 5676$

Old age and RRT criteria are priorities on the checklist. Supplementation with a quantifiable level of severity based on EWS ${ }^{75} 7984$ 91; history of repeat hospitalisations with or without admissions to $\mathrm{ICU}^{95}$; emergency admission ${ }^{96}$; at least one of the predefined advanced comorbidities from the evidence-based list; ${ }^{35}$ an objective measurement of frailty; ${ }^{85}$ documentation of nursing home placement; ${ }^{33} 46$ evidence of cognitive impairment; ${ }^{25} 42637383$ and readily available test results: proteinuria and if ECG confirms abnormalities. $^{97}$

Table 3 shows our resulting 29-item screening tool, named CriSTAL, to denote our intention to introduce transparency in the identification of the dying patient and enable objective clinician decisions about prognosis and

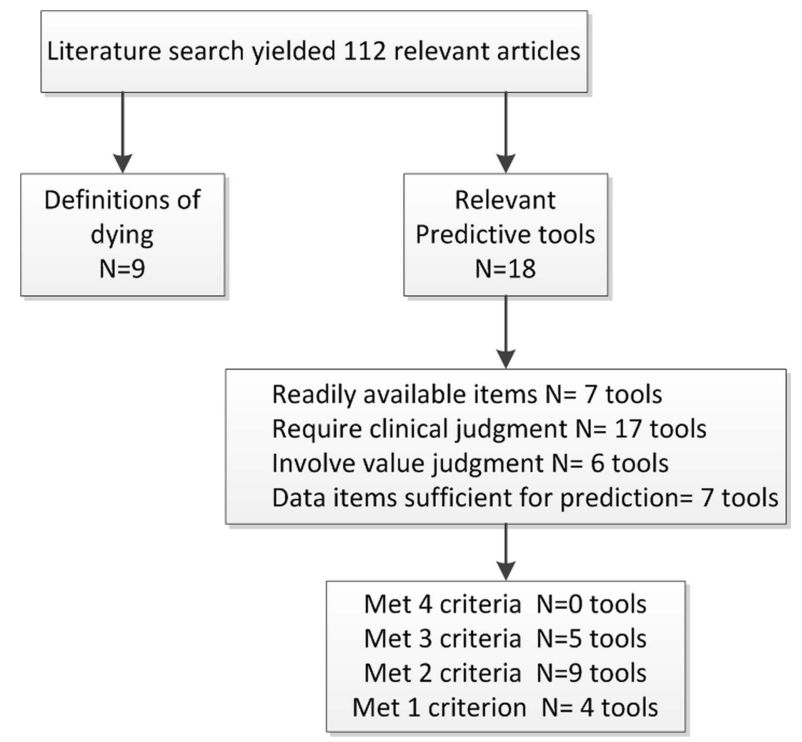

Figure 1 Outcome of the literature review. justification for administering or de-escalating aggressive treatments.

A slight modification is proposed for the use of the tool following a RRT attendance (table 4). This might encourage reassessment of the need for continuing hospitalisation in an acute care facility and discussion about the need for limitations of treatment if death is imminent.

\section{DISCUSSION}

How would CriSTAL be used in practice? It may characterise 'appropriateness of admission' and appropriateness of subsequent treatment for patients at the end of life in a way that can be applicable to a wide variety of terminal health conditions. It could be used as a platform for beginning discussions with patients and their carers. It may also add more certainty identifying the irrevocably dying patient with chronic comorbidities and prevent further futile treatments to prolong life. We have omitted indicators of system failures or nursing staff workloads such as TISS or $\mathrm{NEMS}^{90}$ that may potentially influence risk of death, as these did not fulfil our inclusion criteria of being routinely available

The Scottish health system implemented a national action plan for care at the end of life deriving from the realisation that $30 \%$ of all hospital bed-days were accounted for by multiple admissions of people in the final year of life. $^{41}$ The 'Dying well' premise in Scotland is that alternative care is integral to continuity of care outside the hospital. The strategy includes among others, early identification of care needs for any terminal illness, holistic assessment and involvement of patients and families in the coordination of alternative care. ${ }^{2}$

Inspired by this development, our definition of inappropriate hospital admission is linked to the more objective scoring factors of the CriSTAL tool, whose accuracy is to be validated to more precisely establish the main determinants of death in the short term. Our review indicated that old age $\mathrm{e}^{42638586}$ and concurrent illnesses $^{18} 254246 \quad 63 \quad{ }^{101}$ were the strongest predictors of death in and outside intensive care. ${ }^{45} 104$

Strengths of this developmental work are the evidence-base source of variables in the tool and the extensive range of predictors covering demographic, physiological and diagnostic prediction measures.

A limitation of this research is that the item selection was based on a narrative review with focused set of search terms. This may have led to overlook of some articles that would have been captured in a systematic and broader search strategy. However, the comprehensive search for tools and the breadth of instruments found using this approach provided a sufficiently large number of items to start the discussion on possible amalgamation of variables from existing instruments to meet our targeted need. Other researchers among the readership may choose to 
Table 3 Proposed components of the Criteria for Screening and Triaging to Appropriate aLternative care tool to identify end-of-life status before hospital admission

\begin{tabular}{|c|c|}
\hline$\square$ & Age $\geq 65^{42} 638586$ AND \\
\hline$\square$ & Being admitted via emergency this hospitalisation ${ }^{96}$ (associated with $25 \%$ mortality within 1 year) \\
\hline$\square$ & OR Meets 2 or more of the following deterioration criteria on admission 303298 \\
\hline$\square$ & 1. Decreased LOC: Glasgow Coma Score change $>2$ or AVPU=P or $U$ \\
\hline$\square$ & 2. Systolic blood pressure $<90 \mathrm{~mm} \mathrm{Hg}$ \\
\hline$\square$ & 3. Respiratory rate $<5$ or $>30$ \\
\hline$\square$ & 4. Pulse rate $<40$ or $>140$ \\
\hline$\square$ & 5. Need for oxygen therapy or known oxygen saturation $<90 \%{ }^{33}$ \\
\hline$\square$ & 6. Hypoglycaemia: $\mathrm{BGL}^{99}$ \\
\hline$\square$ & 7. Repeat or prolonged seizures ${ }^{99}$ \\
\hline$\square$ & 8. Low urinary output $(<15 \mathrm{~mL} / \mathrm{h} \text { or }<0.5 \mathrm{~mL} / \mathrm{kg} / \mathrm{h})^{100}$ \\
\hline$\square$ & OR MEW or SEWS score $>4{ }^{46} 79$ \\
\hline AND & OTHER RISK FACTORS /PREDICTORS OF SHORT-MEDIUM-TERM DEATH \\
\hline$\square$ & Personal history of active disease (at least one of): 1825424663101102 \\
\hline$\square$ & Advanced malignancy \\
\hline$\square$ & Chronic kidney disease \\
\hline$\square$ & Chronic heart failure \\
\hline$\square$ & Chronic obstructive pulmonary disease \\
\hline$\square$ & New cerebrovascular disease \\
\hline$\square$ & Myocardial infarction \\
\hline$\square$ & Moderate/severe liver disease \\
\hline$\square$ & Evidence of cognitive impairment (eg, long term mental disorders, dementia, behavioural alterations or disability from stroke) 2542637383 \\
\hline$\square$ & Previous hospitalisation in past year ${ }^{10}$ \\
\hline$\square$ & Repeat ICU admission at previous hospitalisation ${ }^{95}$ (associated with a fourfold increase in mortality) \\
\hline$\square$ & Evidence of frailty: 2 or more of these: 424663858998 \\
\hline$\square$ & Unintentional or unexplained weight loss (10 lbs in past year) ${ }^{18} 8385$ \\
\hline$\square$ & Self-reported exhaustion (felt that everything was an effort or felt could not get going at least 3 days in the past week) ${ }^{85}$ \\
\hline$\square$ & Weakness (low grip strength for writing or handling small objects, difficulty or inability to lift heavy objects $>=4.5 \mathrm{Kg}$ ) ${ }^{63}$ \\
\hline$\square$ & Slow walking speed (walks $4.5 \mathrm{~m}$ in $\geq 7 \mathrm{~s}$ ) \\
\hline$\square$ & Inability for physical activity or new inability to stand ${ }^{46} 98$ \\
\hline$\square$ & Nursing home resident/in supported accommodation 3346 \\
\hline$\square$ & Proteinuria on a spot urine sample: positive marker for chronic kidney disease \& predictor of mortality: >30 mg albumin/g creatinine $e^{56} 103$ \\
\hline$\square$ & Abnormal ECG (Atrial fibrillation, tachycardia, any other abnormal rhythm or $\geq 5$ ectopics/min, Changes to Q or ST waves ${ }^{18} 4297$ \\
\hline
\end{tabular}

expand the search or enhance the tool. In fact, a limitation of CriSTAL's development at this stage is its length for routine administration, and the number of potential predictors which may lead to model 'overfitting'. The testing of too many variables is known to reduce the generalisability of the predictive model. ${ }^{105}$ By retrospective testing and future prospective validation we hope to reduce the total number of items without sacrificing predictive accuracy or generalisability. Initially, CriSTAL's 29 subitems will be tested in a retrospective data review using a case-control study design where cases are all deaths reported from the RRT attendances system in a teaching hospital during 2012-2013. Controls will be age-sex-ward matched records of patients admitted in the same period with an RRT call but did not die before or within 3 months of discharge. Sensitivity, specificity and positive and negative predictive values will be calculated from logistic regression models of matched cases and controls. This retrospective validation has been endorsed by the South Western Sydney Local Health District Ethics Committee. The next step after retrospective testing will be the prospective administration of the validated tool as part of the admissions procedure in emergency and after the RRT calls on general wards.

The accuracy of models with different number of variables will be determined using the area under the receiver-operating characteristics (AUROC) curve. ${ }^{106}$ Minimum accuracy will be defined as area under the ROC curve $\geq 80 \%$, and variables not contributing significantly to the model will be dropped from the instrument. Survival analysis and Cox proportional hazards regression will investigate the most significant predictors of imminent death. A 5\% chance of survival to hospital discharge among those predicted to die will be chosen as the maximum error allowed for the tool to be considered useful. 
Table 4 Proposed components of the Criteria for Screening and Triaging to Appropriate aLternative care tool to identify end-of-life status after a rapid response call where a do-not-resuscitate order is not in place

\begin{tabular}{|c|c|}
\hline$\square$ & Age $\geq 65^{42} 638586$ \\
\hline$\square$ & AND admitted via emergency this hospitalisation ${ }^{96}$ (associated with $25 \%$ mortality within 1 year) \\
\hline$\square$ & OR met 2 or more of the selected MET calling criteria below 303298 \\
\hline$\square$ & 1-Decreased LOC: Glasgow Coma Score change $>2$ or AVPU=P or U \\
\hline$\square$ & 2-Systolic blood pressure $<90 \mathrm{~mm} \mathrm{Hg}$ \\
\hline$\square$ & 3-Respiratory rate $<5$ or $>30$ \\
\hline$\square$ & 4-Pulse rate $<40$ or $>140$ \\
\hline$\square$ & 5-Need for oxygen therapy or known oxygen saturation $<90 \%^{33}$ \\
\hline$\square$ & 6-Hypoglycaemia: BGL ${ }^{99}$ \\
\hline$\square$ & 7-Repeat or prolonged seizures ${ }^{99}$ \\
\hline$\square$ & 8-Low urinary output $(<15 \mathrm{~mL} / \mathrm{h} \text { or }<0.5 \mathrm{~mL} / \mathrm{kg} / \mathrm{h})^{100}$ \\
\hline$\square$ & OR MEW or SEWS score $>4^{46} 79$ \\
\hline AND & OTHER RISK FACTORS /PREDICTORS OF SHORT-MEDIUM-TERM DEATH \\
\hline$\square$ & Personal history of active disease (at least one of): 182542466396101 \\
\hline$\square$ & Advanced malignancy \\
\hline$\square$ & Chronic kidney disease \\
\hline$\square$ & Chronic heart failure, \\
\hline$\square$ & Chronic obstructive pulmonary disease \\
\hline$\square$ & New cerebrovascular disease \\
\hline$\square$ & Myocardial infarction \\
\hline$\square$ & Moderate/severe liver disease \\
\hline$\square$ & Evidence of cognitive impairment (eg, long-term mental disorders, dementia, behavioural alterations or disability from stroke) ${ }^{25} 4263738396$ \\
\hline$\square$ & Length of stay before this RRT call ( $\geq 5$ days predicts 1-year mortality) 4696 \\
\hline $\begin{array}{l}\square \\
\square\end{array}$ & $\begin{array}{l}\text { Previous hospitalisation in past year }{ }^{10} \\
\text { repeat ICU admission at this or previous hospitalisation }{ }^{95} \text { (associated with a fourfold increase in mortality) }\end{array}$ \\
\hline$\square$ & Evidence of frailty: 2 or more of these: 424663858998 \\
\hline$\square$ & Unintentional or unexplained weight loss (10 lbs in past year) ${ }^{18} 8385$ \\
\hline$\square$ & Self-reported exhaustion (felt that everything was an effort or felt could not get going at least 3 days in the past week) ${ }^{85}$ \\
\hline$\square$ & Weakness (low grip strength for writing or handling small objects, difficulty or inability to lift heavy objects $\geq 4.5 \mathrm{~kg})^{63}$ \\
\hline$\square$ & Slow walking speed (walks $4.5 \mathrm{~m}$ in $\geq 7 \mathrm{~s}$ ) \\
\hline$\square$ & Inability for physical activity or new inability to stand ${ }^{46} 98$ \\
\hline$\square$ & Nursing home resident/in supported accommodation 334696 \\
\hline$\square$ & Proteinuria on a spot urine sample: positive marker for chronic kidney disease \& predictor of mortality: >30 mg albumin/g creatinine $\mathrm{e}^{56} 103$ \\
\hline$\square$ & Abnormal ECG (Atrial fibrillation, tachycardia, any other abnormal rhythm or $\geq 5$ ectopics/min, Changes to Q or ST waves ${ }^{18} 4297$ \\
\hline
\end{tabular}

MET, medical emergency team; MEW, modified early warning; RRT, rapid response team.

While it is acknowledged that predictions based on population subgroups are not meant to be used for individuals, ${ }^{107}$ the calculated risk can be used as a reference to inform the decision by the individual under the clinician's guidance, on whether or not to continue aggressive treatment, given the odds of dying based on the well-established predictors. Careful use of the CriSTAL tool care for decision-making would involve alignment with quality of care principles and patient values and preferences, and should not be driven by hospital financial pressures or need to meet health system performance indicators. ${ }^{24}$

Finally, it is important to recognise that the use of a screening tool for identifying patients who have a high probability of dying within 3 months can only provide an indication of those who with a low probability of survival and will not be a signal of absolute certainty. ${ }^{50}$ Testing its appropriateness, reliability and predictive value in different patient subpopulations will help reduce this uncertainty but its predictive value may vary in different settings and for different timeframes and this needs to be ascertained. Further, its values after an RRT response will need to be assessed in relation to its value at the time of admission for patients when trialled. As emphasised before, testing in different settings could yield different predictive performance depending on the patient profile and possibly the influence of factors not accounted for in the tool. Readers and researchers are encouraged to train and validate the CriSTAL tool in their facility to generate the most valid and relevant set of variables for their subpopulations.

\section{CONCLUSIONS AND RECOMMENDATIONS}

This tool does not intend to preclude access to healthcare for the terminal elderly, but to provide an 
objective assessment and definition of the dying patient as a starting point for honest communication with patients and families, ${ }^{3}$ about recognising that dying is part of the life cycle. Dignified withdrawal of intensive and inappropriate treatment ${ }^{29} 52$ and triage into alternative care in non-acute facilities ${ }^{10}{ }^{38}$ is an area where there is still ample room for improvement. ${ }^{1} 2939$ Standard guidelines for alternative end-of-life care are not yet broadly adopted in Australia and discussions with policy-makers need to continue. ${ }^{2}$ However, increasing evidence of alternative out-of-hospital care acceptable to clinicians ${ }^{108}$ and others are known to include sedation to minimise distress, pain management, ${ }^{109}$ spiritual support, ${ }^{41}$ music therapy and home-based palliative care. ${ }^{110}$ If proven accurate in the prediction of short-term death, a reduced version of CriSTAL could be proposed for routine use at hospital admission. We acknowledge that the Australian health system may not yet be equipped to respond to the demand for alternative healthcare facilities for the dying. ${ }^{111}$ However, it is hoped that using such predictive tools may encourage more appropriate services for managing patients at the end of life.

Training for nurses and doctors in the use of the screening tool and in approaching patients and families with concrete information about inevitability of death and lack of benefit of further intensive treatment are paramount. ${ }^{27} 112$ They will be better equipped to communicate the responsible decision to suspend efforts and handle potential requests for futile treatment. ${ }^{4149}$

Automation of CriSTAL and its scoring would facilitate use at time of admission and production of instant or retrospective locally relevant profiles of patients imminently dying. Potential uses include as a clinical support tool for decision-making on triage to appropriate end-of-life care facilities; to prevent death in some cases; and to examine variation in risk-of-death levels, differences in admission practices, and inform triage policies across hospitals, ${ }^{43}$ as a first step into cost-effectiveness and patient satisfaction studies.

Contributors MCM conceived the idea of an objective assessment. KH and MCM led the literature searches. MCM summarised findings, wrote the first and final draft manuscript and refined all versions. $\mathrm{KH}$ contributed to the literature review, provided ongoing intellectual input and revised subsequent manuscript drafts.

Funding This article presents independent research funded by a grant from the National Health and Medical Research Council of Australia (\# 1054146).

Competing interests $\mathrm{KH}$ is an advocate for dying with dignity and has written books and articles on the subject.

Provenance and peer review Not commissioned; externally peer reviewed.

Data sharing statement No additional unpublished data exists from this study. A retrospective validation of the screening tool is in preparation. Protocols and materials for the conduct of that validation could be shared with other research groups interested in testing the screening tool in their patient populations.

Open Access This is an Open Access article distributed in accordance with the Creative Commons Attribution Non Commercial (CC BY-NC 4.0) license, which permits others to distribute, remix, adapt, build upon this work noncommercially, and license their derivative works on different terms, provided the original work is properly cited and the use is non-commercial. See: http://creativecommons.org/licenses/by$\mathrm{nc} / 4.0$ /

\section{REFERENCES}

1 Tsim S, Davidson S. End-of-life care in a general respiratory ward in the United Kingdom. Am J Hosp Palliat Med 2014;31:172-4.

2 The Scottish Government. Living and dying well: building on progress. Secondary living and dying well: building on progress 2011. http://www.scotland.gov.uk/Resource/Doc/ 340076/0112559.pdf

3 Hillman K. Dying safely. Int J Qual Health Care 2010;22:339-40.

4 Higginson IJ, Sen-Gupta GJ. Place of care in advanced cancer: a qualitative systematic literature review of patient preferences. J Palliat Med 2000;3:287-300.

5 Beccaro M, Costantini M, Giorgi Rossi P, et al. Actual and preferred place of death of cancer patients. Results from the Italian survey of the dying of cancer (ISDOC). J Epidemiol Community Health 2006;60:412-16.

6 Brazil K, Howell D, Bedard M, et al. Preferences for place of care and place of death among informal caregivers of the terminally ill. Palliat Med 2005;19:492-9.

7 Cohen J, Bilsen J, Addington-Hall J, et al. Population-based study of dying in hospital in six European countries. Palliat Med 2008;22:702-10.

8 Wilson DM, Truman CD, Thomas R, et al. The rapidly changing location of death in Canada, 1994-2004. Soc Sci Med 2009;68:1752-8.

9 Lowthian JA, Jolley DJ, Curtis AJ, et al. The challenges of population ageing: accelerating demand for emergency ambulance services by older patients, 1995-2015. Med J Aust 2011;194:574-8.

10 Rosenwax LK, McNamara BA, Kevin Murray K, et al. Hospital and emergency department use in the last year of life: a baseline for future modifications to end-of-life care. Med J Aust 2011;194:570-73.

11 Hillman K. The changing role of acute-care hospitals. Med J Aust 1999;170:325-28.

12 Earle CC, Landrum MB, Souza JM, et al. Aggressiveness of cancer care near the end of life: is it a quality-of-care issue? J Clin Oncol 2008;26:3860-6.

13 Earle CC, Park ER, Lai B, et al. Identifying potential indicators of the quality of end-of-life cancer care from administrative data. J Clin Oncol 2003;21:1133-8.

14 Senthuran S, Bandeshe H, Ranganathan D, et al. Outcomes for dialysis patients with end-stage renal failure admitted to an intensive care unit or high dependency unit. Med J Aust 2008;188:292-5.

15 Stolman CJ, Gregory JJ, Dunn D, et al. Evaluation of the do not resuscitate orders at a community hospital. Arch Intern Med 1989;149:1851-6.

16 Weissman D. Policy Forum. Policy Proposal: Do Not Resuscitate Orders: A Call for Reform. Virtual Mentor [Internet]. 2003 [cited 2014 August]; 5(1). Available from: http://virtualmentor.ama-assn.org/2003/01/pfor2-0301.html 
17 Holloway RG, Benesch CG, Burgin WS, et al. Prognosis and decision making in severe stroke. JAMA 2005;294: 725-33.

18 van Walraven C, Austin PC, Jennings A, et al. A modification of the Elixhauser comorbidity measures into a point system for hospital death using administrative data. Med Care 2009;47:626-33.

19 Phillips G. Do not resuscitate orders: a reappraisal. HEC Forum 1990;2:101-4.

20 Wright AA, Zhang B, Ray A, et al. Associations between end-of-life discussions, patient mental health, medical care near death, and caregiver bereavement adjustment. JAMA 2008;300:1665-73.

21 National Health Service. End of Life Care Strategy. Promoting high quality care for all adults at the end of life. July. Secondary End of Life Care Strategy. Promoting high quality care for all adults at the end of life. July 2008. http:// webarchive.nationalarchives.gov.uk/20130107105354/http:// www.dh.gov.uk/prod_consum_dh/groups/dh_digitalassets/@ dh/@en/documents/digitalasset/dh_086345.pdf

22 van Weel C, Michels J. Dying, not old age, to blame for costs of health care. Lancet 1997;350:1159-60.

23 Curtis JR, Engelberg RA, Bensink ME, et al. End-of-life care in the intensive care unit. Can we simultaneously increase quality and reduce costs? Am J Respir Crit Care Med 2012;186:587-92.

24 Oshima Lee E, Emanuel EJ. Shared decision making to improve care and reduce costs. N Engl J Med 2013;368: 6-8.

25 Downar J, Rodin D, Barua R, et al. Rapid response teams, do not resuscitate orders, and potential opportunities to improve end-of-life care: a multicentre retrospective study. J Crit Care 2013;28:498-503.

26 Gleeson K. The do-not-resuscitate order: still too little too late. Arch Intern Med 1990;150:1057-60.

27 Mead GE, Turnbull CJ. Cardiopulmonary resuscitation in the elderly: patients' and relatives' views. J Med Ethics 1995;21:39-44.

28 Heyland DK, Dodek P, Rocker G, et al. What matters most in end-of-life care: perceptions of seriously ill patients and their family members. CMAJ 2006;174:627-33.

29 Kompanje EJ, Piers RD, Benoit DD. Causes and consequences of disproportionate care in intensive care medicine. Curr Opin Crit Care 2013;19:630-5.

30 Hillman $\mathrm{K}$, Chen J, Cretikos M, et al. Introduction of the medical emergency team (MET) system: a cluster-randomised controlled trial. Lancet 2005;24:2091-7.

31 Mitchell IA, McKay H, Van Leuvan C, et al. A prospective controlled trial of the effect of a multi-faceted intervention on early recognition and intervention in deteriorating hospital patients. Resuscitation 2010;81:658-66.

32 Goldhill DR, McNarry AF. Physiological abnormalities in early warning scores are related to mortality in adult inpatients†. Br J Anaesth 2004;92:882-84.

33 Jones DA, Bagshaw SM, Barrett J, et al. The role of the medical emergency team in end-of-life care: a multicenter, prospective, observational study. Crit Care Med 2012;40:98-103.

34 Jones D, Moran J, Winters B, et al. The rapid response system and end-of-life care. Curr Opin Crit Care 2013;19:616-23.

35 Jones DA, McIntyre T, Baldwin I, et al. The medical emergency team and end-of-life care: a pilot study. Crit Care Resusc 2007;9:151-56.
36 Stacey D, Legare F, Col NF, et al. Decision aids for people facing health treatment or screening decisions. Cochrane Database Syst Rev 2014;1: CD001431.

37 van Walraven C, Forster AJ, Stiell IG. Derivation of a clinical decision rule for the discontinuation of in-hospital cardiac arrest resuscitations. Ann Intern Med 1999;159:129-34.

38 Kennedy C, Brooks-Young P, Brunton Gray C, et al. Diagnosing dying: an integrative literature review. $B M J$ Support Palliat Care 2014;4:263-70.

39 Curtis JR, Engelberg RA, Nielsen EL, et al. Patient-physician communication about end-of-life care for patients with severe COPD. Eur Respir J 2004;24:200-5.

40 Gomes B, Higginson IJ. Home or hospital? Choices at the end of life. J Royal Soc Med 2004;97:413-14.

41 Paterson BC, Duncan R, Conway R, et al. Introduction of the Liverpool Care Pathway for end of life care to emergency medicine. Emerg Med J 2009;26:777-9.

42 Viganò A, Dorgan M, Buckingham J, et al. Survival prediction in terminal cancer patients: a systematic review of the medical literature. Palliat Med 2000;14:363-74.

43 Escobar GJ, Greene JD, Scheirer P, et al. Risk-adjusting hospital inpatient mortality using automated inpatient, outpatient, and laboratory databases. Med Care 2008;46:232-9.

44 Rothman SI, Rothman MJ, Solinger AB. Placing clinical variables on a common linear scale of empirically based risk as a step towards construction of a general patient acuity score from the electronic health record: a modelling study. BMJ Open 2013;13:e002367.

45 Wang CY, Calfee CS, Paul DW, et al. One-year mortality and predictors of death among hospital survivors of acute respiratory distress syndrome. Intensive Care Med 2014;40:388-96.

46 Kellett J, Deane B. The Simple Clinical Score predicts mortality for 30 days after admission to an acute medical unit. QJM 2006;99:771-81.

47 Sharif N, Irfan M, Hussain J, et al. Factors Associated within 28 Days In-Hospital Mortality of Patients with Acute Respiratory Distress Syndrome BioMed Research International 2013; Article ID 564547:5 pages doi: http://dx.doi.org/10. 1155/2013/564547 [published Online First: Epub Date]|.

48 President's Commission for the Study of Ethical Problems in Medicine and Biomedical and Behavioral Research. Defining death. medical, legal and ethical issues in the determination of death. 1981 https://bioethicsarchive.georgetown.edu/pcbe/ reports/past_commissions/defining_death.pdf (accessed Mar 2013).

49 Blackhall LJ. Must we always use CPR? New England J Med 1987;317:1281-5.

50 Tomlinson T, Brody H. Futility and the ethics of resuscitation. JAMA 1990;264:1276-80.

51 Australian Government Department of Health and Ageing. Guidelines for a Palliative Approach in Residential Aged Care. Secondary Guidelines for a Palliative Approach in Residential Aged Care 2006. http://www.nhmrc.gov.au/_files_nhmrc/ publications/attachments/pc29.pdf

52 Schmidt RJ, Moss AH. Dying on dialysis: the case for a dignified withdrawal. Clin J Am Soc Nephrol 2014;9:174-80.

53 Clark A, Fallowfield LJ. Quality of life measures in patients with malignant disease. J Royal Soc Med 1986;79:165-69.

54 Addington-Hall MacDonald, LD Anderson HR. Can the Spitzer quality of life index help to reduce prognostic uncertainty in terminal care? Br J Cancer 1990;62:695-99. 
55 Péus D, Newcomb N, Hofer S. Appraisal of the Karnofsky performance status and proposal of a simple algorithmic system for its evaluation. BMC Med Inform Decis Mak 2013;13:72.

56 Rothman MJ, Solinger AB, Rothman SI, et al. Clinical implications and validity of nursing assessments: a longitudinal measure of patient condition from analysis of the Electronic Medical Record. BMJ Open 2012;2: pii: e000646

57 Fromme EK, Smith MD, Bascom PB, et al. Incorporating routine survival prediction in a US hospital-based palliative care service. J Palliat Med 2010;13:1439-44.

58 Rockwood K, Song X, MacKnight C, et al. A global clinical measure of fitness and frailty in elderly people. CMAJ 2005;173:489-5.

59 Weiche RE, Mundy BJ, Skokan L, et al. Patient-centered research. Identifying patients nearing the end of life from congestive heart failure or chronic obstructive pulmonary disease. J Gen Intern Med 2000;15(Suppl 2):9-10.

60 Mossey JM, Shapiro E. Self-rated health: a predictor of mortality among the elderly. Am J Public Health 1982;72:800-8.

61 Jylhä M. What is self-rated health and why does it predict mortality? Towards a unified conceptual model. Soc Sci Med 2009;69:307-16.

62 Heistaro S, Jousilahti P, Lahelma E, et al. Self rated health and mortality: a long term prospective study in eastern Finland. J Epidemiol Community Health 2001;55:227-32.

63 Saliba D, Elliott M, Rubenstein LZ, et al. The vulnerable elders survey: a tool for identifying vulnerable older people in the community. J Am Geriatr Soc 2001;49:1691-9.

64 Knaus WA, Draper EA, Wagner DP, et al. APACHE II: a severity of disease classification system. Crit Care Med 1985;13:818-29.

65 McMahon LF Jr, Hayward RA, Bernard AM, et al. APACHE-L: a new severity of illness adjuster for inpatient medical care. Med Care 1992;30:445-2.

66 Del Bufalo C, Morelli A, Bassein L, et al. Severity scores in respiratory intensive care: APACHE II predicted mortality better than SAPS II. Respir Care 1995;40:1042-7.

67 Zimmerman JE, Kramer AA, McNair DS, et al. Acute Physiology and Chronic Health Evaluation (APACHE) IV: hospital mortality assessment for today's critically ill patients. Crit Care Med 2006;34:1297-310.

68 Charlson ME, Pompei P, Ales KL, et al. A new method of classifying prognostic comorbidity in longitudinal studies: development and validation. J Chronic Dis 1987;40:373-83.

69 Pompei P, Charlson ME, Gordon Douglas R Jr. Clinical assessments as predictors of one year survival after hospitalization: Implications for prognostic stratification. J Clin Epidemiol 1988;41:275-84.

70 Le Gall J, Lemeshow S, Saulnier F. A new simplified acute physiology score (saps ii) based on a European/north American multicenter study. JAMA 1993;270:2957-63.

71 Anderson F, Downing GM, Hill J, et al. Palliative performance scale (PPS): a new tool. J Palliat Care 1996;12:5-11.

72 Virik K, Glare P. Validation of the Palliative Performance Scale for Inpatients admitted to a palliative care unit in Sydney, Australia. J Pain Symptom Manage 2002;23:455-7.

73 Elixhauser A, Steiner C, Harris DR, et al. Comorbidity measures for use with administrative data. Med Care 1998;36:8-27.
74 Austin SR, Wong YN, Uzzo RG, et al. Why summary comorbidity measures such as the Charlson comorbidity index and Elixhauser score work. Med Care 2013. Published Online First (May 23) doi:10.1097/MLR.0b013e318297429c

75 Subbe CP, Kruger M, Rutherford P, et al. Validation of a modified early warning score in medical admissions. QJM 2001;94:521-26.

76 Glare PA, Eychmueller S, McMahon P. Diagnostic accuracy of the palliative prognostic score in hospitalized patients with advanced cancer. J Clin Oncol 2004;22:4823-8.

77 Maltoni M, Scarpi E, Pittureri C, et al. Prospective comparison of prognostic scores in palliative care cancer populations. Oncologist 2012;17:446-54.

78 Paterson R, MacLeod DC, Thetford D, et al. Prediction of in-hospital mortality and length of stay using an early warning scoring system: clinical audit. Clin Med 2006;6:281-84.

79 Kuo S-H, Tsai C-F, Li C-R, et al. Rapid emergency medicine score as a main predictor of mortality in Vibrio vulnificusrelated patients. Am J Emerg Med 2013;31:1037-41.

80 Kellett J, Rasool S, McLoughlin B. Prediction of mortality 1 year after hospital admission. QJM 2012;105:847-53.

81 Groarke JD, Gallagher J, Stack J, et al. Use of an admission early warning score to predict patient morbidity and mortality and treatment success. Emerg Med J 2008;25:803-6.

82 Stone CA, Tiernan E, Dooley BA. Prospective validation of the palliative prognostic index in patients with cancer. J Pain Symptom Manage 2008;35:617-22.

83 Glare P, Sinclair C, Downing M, et al. Predicting survival in patients with advanced disease. Eur J Cancer 2008;44:1146-56.

84 Prytherch DR, Smith GB, Schmidt PE, et al. ViEWSTowards a national early warning score for detecting adult inpatient deterioration. Resuscitation 2010;81:932-7.

85 Fried LP, Tangen CM, Walston J, et al. Frailty in older adults: evidence for a phenotype. J Gerontol A Biol Sci Med Sci 2001;56:M146-57.

86 Hucker TR, Mitchell GP, Blake LD, et al. Identifying the sick: can biochemical measurements be used to aid decision making on presentation to the accident and emergency department. Br J Anaesth 2005;94:735-41.

87 Alloway L, Minton O. The accuracy of the clinical prediction of survival: a comparison of doctors' and nurses' estimations and the failure to validate the Palliative Prognostic Score [abstract for the 5th palliative care congress, UK]. Palliat Med 2004;18:155.

88 Lübke T, Mönig SP, Schneider PM, et al. Does Charlson-comorbidity index correlate with short-term outcome in patients with hastric cancer? [Article in German]. Zentralbl Chir 2003;128:970-6.

89 Le Maguet P, Roquilly A, Lasocki S, et al. Prevalence and impact of frailty on mortality in elderly ICU patients: a prospective, multicenter, observational study. Intensive Care Med 2014:1-9.

90 Vincent JL, Moreno R. Clinical review: scoring systems in the critically ill. Crit Care 2010;14:207.

91 Hands C, Reid E, Meredith P, et al. Patterns in the recording of vital signs and early warning scores: compliance with a clinical escalation protocol. BMJ Qual Saf 2013;22:719-26.

92 Moon A, Cosgrove JF, Lea D, et al. An eight year audit before and after the introduction of modified early warning score (MEWS) charts, of patients admitted to a tertiary referral intensive care unit after CPR. Resuscitation 2011;82:150-54.

93 Parham G. Recognition and response to the clinically deteriorating patient. MJA 2012;3:18-22. 
94 Morgan RJM, Wright MM. In defence of early warning scores. Br J Anaesth 2007;99:747-48.

95 Kramer AA, Higgins TL, Zimmerman JE. Intensive care unit readmissions in US hospitals: patient characteristics, risk factors, and outcomes. Crit Care Med 2012;40:3-10.

96 Levine SK, Sachs GA, Jin L, et al. A prognostic model for 1-year mortality in older adults after hospital discharge. Am J Med 2007;120:455-60.

97 Copeland GP, Jones D, Walters M. POSSUM: a scoring system for surgical audit. Br J Surg 1991;78:355-60.

98 DeVita MA, Smith GB, Adam SK, et al. "Identifying the hospitalised patient in crisis"-a consensus conference on the afferent limb of Rapid Response Systems. Resuscitation 2010;81:375-82. http://dx.doi.org/10.1016/j.resuscitation. 2009.12.008

99 Cretikos M, Parr M, Hillman K, et al. Guidelines for the uniform reporting of data for Medical Emergency Teams. Resuscitation 2006;68:11-25.

100 Cerda J. Oliguria: an earlier and accurate biomarker of acute kidney injury [quest]. Kidney Int 2011;80:699-701.

101 Krumholz HM, Merrill AR, Schone EM, et al. Patterns of hospital performance in acute myocardial infarction and heart failure 30-day mortality and readmission. Circ Cardiovasc Qual Outcomes 2009;2:407-13.

102 Knaus WA, Harrell FE Jr, Lynn J, et al. The SUPPORT prognostic model. Objective estimates of survival for seriously ill hospitalized adults. Study to understand prognoses and preferences for outcomes and risks of treatments. Ann Intern Med 1995;122:191-203.

103 Peralta CA, Shlipak MG, Judd S, et al. Detection of chronic kidney disease with creatinine, cystatin $\mathrm{c}$, and urine albumin-to-creatinine ratio and association with progression to end-stage renal disease and mortality. JAMA 2011;305:1545-52.

104 Kaukonen K, Bailey M, Suzuki S, et al. Mortality related to severe sepsis and septic shock among critically ill patients in Australia and New Zealand, 2000-2012. JAMA 2014;311:1308-16.

105 Lynn J, Teno JM, Harrell FE Jr. Accurate prognostications of death. Opportunities and challenges for clinicians. West J Med 1995;163:250-7.

106 Fawcett T. An introduction to ROC analysis. Pattern Recognit Lett 2006;27:861-74.

107 Brabrand M, Folkestad L, Clausen NG, et al. Risk scoring systems for adults admitted to the emergency department: a systematic review. Scand J Trauma Resusc Emerg Med 2010;18:1-8.

108 Simon ST, Martens M, Sachse M, et al. Liverpool Care Pathway" (LCP) in Deutschland. Care of the dying in hospital: initial experience with the Liverpool Care Pathway (LCP) in Germany. Dtsch Med Wochenschr 2009;134:1399-404.

109 Waldrop DP, Kirkendall AM. Comfort measures: a qualitative study of nursing home-based end-of-life care. J Palliat Med 2009;12:719-24.

110 Ryder-Lewis M. Going home from ICU to die: a celebration of life*. Nurs Crit Care 2005;10:116-21.

111 Horey DE, Street AF, Sands AF. Acceptability and feasibility of end-of-life care pathways in Australian residential aged care facilities. Med J Aust 2012;197:106-9.

112 Cherniack E. Increasing use of DNR orders in the elderly worldwide: whose choice is it? J Med Ethics 2002;28:303-07. 